Trans

continentales

\title{
Transcontinentales
}

Sociétés, idéologies, système mondial

\section{Pourquoi ne pas parler d'exception sanitaire?}

Entretien conduit par Jean-Luc Racine

Why not to recognize the health exception? Interview with Rony Brauman

\section{Rony Brauman}

\section{CpenEdition}

\section{Journals}

Édition électronique

URL : http://journals.openedition.org/transcontinentales/738

DOI : 10.4000/transcontinentales.738

ISBN : 978-2-7351-1563-1

ISSN : 1775-397X

Éditeur

Editions de la maison des sciences de l'homme

\section{Édition imprimée}

Date de publication : 31 décembre 2007

Pagination : 113-119

ISBN : 978-2-200-92397-6

ISSN : $1950-1684$

\section{Référence électronique}

Rony Brauman, «Pourquoi ne pas parler d'exception sanitaire? », Transcontinentales [En ligne], 5| 2007, document 7, mis en ligne le 15 avril 2011, consulté le 08 septembre 2020. URL : http:// journals.openedition.org/transcontinentales/738; DOI : https://doi.org/10.4000/transcontinentales. 738 


\title{
Entretien avec Rony Brauman \\ Pourquoi ne pas parler d'exception sanitaire?
}

\begin{abstract}
Rony Brauman est médecin (médecine tropicale, santé publique et épidémiologie). À compter de 1978, il intervient en Asie, en Afrique et en Amérique centrale dans le cadre de l'assistance mise en ceuvre par Médecins sans frontières, organisation dont il assure la présidence de 1982 à 1994. Il est actuellement directeur de recherches à la Fondation Médecins sans frontières et professeur associé à l'Institut d'études politiques de Paris. Figure emblématique de l'action humanitaire, dont il sait aussi critiquer les ambiguités, il a publié, entre autres, L'action humanitaire (Flammarion, 1993), Humanitaire, le dilemme (avec Philippe Petit, Textuel, 2007), Utopies sanitaires (le Pommier, 2000), Aider, sauver : pourquoi, comment? (Bayard, 2006), et Penser dans l'urgence: parcours critique d'un humanitaire (avec Catherine Portevin, Le Seuil, 2006).
\end{abstract}

Transcontinentales l'a interrogé sur la fracture sanitaire entre pays du Nord et pays du Sud, sur les rapports entre santé et marché, au crible de la transformation de la pratique du brevet, sur l'extension du concept de crise sanitaire et sur les aléas du principe de précaution.

Transcontinentales : Comme bien d'autres, Médecins sans frontières a tiré la sonnette d'alarme en raison du déclin des recherches portant sur les maladies tropicales, dont la part baisse constamment par rapport à l'ensemble des recherches sur de nouveaux médicaments. Sont en cause en l'affaire, le rapport de la santé au marché, la question des droits de propriété intellectuelle et les stratégies des grandes firmes pharmaceutiques. Jusqu'à quel point l'argument selon lequel le profit est indispensable pour financer la recherche vous paraît-il recevable?

Rony Brauman: Avant d'aborder ce point précis, il est bon de se souvenir que lorsque les médicaments appropriés aux pathologies tropicales ont été mis au point, c'était dans un contexte où ils étaient utiles au système sociopolitique dans lequel ils apparaissaient. C'était une nécessité impériale, coloniale, ou immédiatement postérieure à la colonisation. Il est frappant, par exemple, de constater que la recherche sur le paludisme a marqué le pas à partir du milieu des années 1975, quand les Américains se sont retirés du Vietnam. Jusqu'alors, la recherche en ce domaine était très active. Il en fut de même pour la maladie du sommeil, la leishmaniose, et d'autres grandes endémies spécifiques au milieu tropical. D'une certaine manière, on comprend que le déclin de ces recherches ait coïncidé avec le retrait territorial des pouvoirs impériaux qui effectivement avaient eu un grand besoin de ces médicaments : rappelons que le vaccin contre la fièvre jaune a permis, au XIX ${ }^{e}$ siècle, la pénétration européenne à l'intérieur du continent 
africain, et que c'est bien une perspective conquérante qui avait encouragé la recherche de ce vaccin. Selon la même logique, le retrait impérial a été le prélude au retrait de la recherche clinique dans ce domaine-là.

TC : Ce qui veut dire que les pays nouvellement indépendants n'ont pas eu les moyens de mener des recherches autonomes?

R.B.: En effet, les pays nouvellement indépendants n'ont eu ni les moyens ni vraiment la volonté, en tout cas jusqu'à récemment, de conduire des recherches. On voit toutefois apparaitre cette volonté en Chine et en Inde en particulier, mais également en Thaïlande et dans d'autres pays asiatiques. Aujourd'hui l'Inde est, je crois, le quatrième producteur de médicaments dans le monde. Quant à la Chine, elle est arrivée sur le marché avec un médicament contre le paludisme, mais il semble que d'autres produits doivent suivre. Le déséquilibre post-colonial est en train d'être partiellement rattrapé, en tout cas en Asie. En Afrique, c'est moins vrai. Certes, au Maghreb ou en Afrique du Sud, des potentiels de recherche commencent à s'exprimer, mais on est encore très, très loin du compte. Pour ce qui est des brevets, il faut, là encore, replacer la question dans son contexte historique. Pendant très longtemps, on ne déposait pas de brevets et la recherche clinique progressait tout de même. L'argument selon lequel des brevets, et donc la propriété intellectuelle, seraient la condition sine qua non de la recherche clinique doit donc être modéré. Je dis simplement «modéré» parce que je crois que l'argument recèle une part de légitimité, et c'est là que la question, évidemment, se complique. Historiquement, une bonne partie de la recherche clinique s'est faite sans brevet, mais aussi sans méthodologie de mise à l'épreuve comparable aux essais cliniques en double-aveugle qui sont aujourd'hui la règle intangible. L'essai en doubleaveugle impose de mettre en lice deux produits - le médicament testé et un placebo ou un médicament existant - sans que le médecin ni le patient ne sachent quel est le bon produit.

TC : Si la recherche médicale a pu se faire longtemps sans brevet, tout en continuant à innover, est-ce parce que les travaux étaient conduits essentiellement par des laboratoires publics, ou pas nécessairement?

R.B. : On trouvait à la fois des laboratoires publics et des laboratoires privés. Le laboratoire Winthrop par exemple, absorbé aujourd'hui dans le groupe Sanofi-Aventis, a joué un rôle important dans la recherche tropicale et même si la recherche publique est très importante, la recherche privée a contribué également aux avancées dans le domaine.

\section{TC : Et aujourd'hui?}

R.B. : Aujourd'hui le contexte économique et scientifique a changé. De très nombreuses innovations médicales ont vu le jour et, semble-t-il, depuis vingt ou trente ans, on peine à en réaliser d'autres. La question des brevets se pose donc de façon différente. Le problème n'est pas tant le brevet en soi que l'usage qui en est fait. Les brevets ont été créés pour protéger l'innovation contre le pillage des 
avancées de la recherche, mais ils sont aujourd'hui utilisés pour installer des monopoles de fait en matière de commercialisation des médicaments. On constate à la fois une évolution de l'esprit dans lequel les brevets sont déposés et une évolution du concept de ce qui est brevetable. La tendance aujourd'hui est de tout breveter, pas seulement le résultat, mais aussi des mots, des gènes, des façons de faire...

TC : C'est le débat à l'Organisation mondiale du commerce (OMC) sur la distinction entre le produit et le procédé par lequel on fabrique le produit.

R.B. : Oui, mais pas seulement. Les matières qui sont utilisées et qui entrent dans le procédé, puis les étapes du procédé, sont aussi brevetées. Un verrouillage très serré s'est donc installé, avec l'extension du domaine du brevetage, jusqu'au brevetage du vivant, qui est en soi un sujet très préoccupant.

Une des raisons, et peut-être même la raison primordiale, pour laquelle les grands laboratoires se sont jetés dans la bataille contre les génériques pour défendre leurs droits de propriété intellectuelle, c'est précisément parce qu'ils peinaient à innover. Prenez par exemple Pfizer, le numéro un mondial, qui dépasse de près de 50 \% le numéro deux. Il est d'une puissance phénoménale, mais il n’a pas sorti un seul médicament innovant au cours de ces dix dernières années et son seul blockbuster innovant a été le Viagra, dont on ne peut dire qu'il soit un médicament décisif pour la santé publique. Qui plus est, Pfizer n'a pas «inventé» le Viagra, il a racheté le laboratoire qui l'a fabriqué. Voilà ce qu'on appelle de la recherche et du brevet! Dans ces conditions, les laboratoires ont encore du travail à faire avant de démontrer que les brevets n'offrent pas simplement l'opportunité d'installer une économie de rente alors même qu'ils prétendent générer une économie d'innovation permanente. Il y a quand même quelque audace à prétendre que "plus on brevette plus on innove», alors qu'on n'a cessé de breveter de manière croissante ces vingt dernières années, tandis qu'on voyait dans le même temps l'innovation décroître. Il ne faut pas méconnaître l'acharnement que mettent les laboratoires à peser sur les politiques de santé par leurs lobbies, par leurs réseaux d'influences, mais aussi par leurs réseaux de formation. En France par exemple, la quasi-totalité de la formation post-universitaire des médecins est assurée par des laboratoires pharmaceutiques. On ne peut pas considérer, dans ces conditions, qu'il s'agisse de formation scientifique permanente. C'est plutôt du marketing sophistiqué, et l'on va inévitablement au devant de lourds problèmes si l'on continue ainsi. Il ne s'agit pas de vouer à l'opprobre ou de stigmatiser ces laboratoires, il est naturel qu'ils se fassent entendre puisque ce sont des acteurs, et donc des interlocuteurs du monde de la santé. Mais il faut donner aux structures publiques les moyens de résister à l'influence financière des grands groupes pharmaceutiques et aux tentations qu'elle peut induire.

TC : On met à l'affiche les objectifs du Millénaire et l'on fait campagne pour l'accès des pays pauvres aux médicaments essentiels. Comment repenser les politiques de développement au sens large, et les modes d'intervention concrets dans le domaine sanitaire? Pour poser le problème autrement, le vieux slogan de «la santé pour tous» peut-il être autre chose qu'un slogan? 
R.B. : Je ne crois pas que ce puisse être autre chose qu'un slogan. D'ailleurs, dans une telle formule, qu'entend-on par santé? Trop souvent prévaut dans ce genre de discours une approche très idéologique, une sorte d'hygiénisme envahissant qui définit la santé comme un état de complet bien-être physique, psychologique et social, ce qui est, à proprement parler, une illusion. Il est plus pertinent de penser la santé en termes d'accès aux soins - et à des soins de qualité - et $\mathrm{d}^{\prime}$ adopter une philosophie disons canguilhemienne ${ }^{1}$, pour laquelle la santé, c'est aussi la possibilité de tomber malade et de s'en relever. Ce point de vue-là me paraît plus opérationnel, et il ouvre des perspectives de coopération. Laissons de côté les utopies, les slogans, les grands programmes qui ne sont pas toujours crédibles, y compris les objectifs du Millénaire, pour parler de la coopération en matière de santé et en matière d'éducation, les deux domaines les plus prometteurs et les plus importants pour tous, et qui sont aussi des champs dans lesquels une aide véritablement efficace peut s'installer. On le voit du reste aujourd'hui : la formation des médecins dans de très nombreux pays dits «du Tiers Monde» est déjà une réalité, y compris en Afrique, ce que l'on l'ignore souvent dans nos représentations convenues. Les équipes d'aide humanitaire médicale [...] s'appuient très largement sur les équipes médicales locales des pays dans lesquels elles travaillent, non seulement parce que ces équipes sont là et qu'il faut coopérer, mais bien parce qu'elles disposent des savoir-faire, des compétences et de la motivation nécessaires.

TC : La plaidoirie d'Amartya Sen formulée de longue date pour investir avant toute chose dans la santé et dans l'éducation commencerait-elle à être entendue?

R.B. : Elle commence à être entendue, même si le résultat n'est pas immédiatement visible parce qu'il est oblitéré par d'autres images qui viennent le parasiter. De fait, il n'y a pas assez de médecins en Afrique. Mais il y en a infiniment plus que quand j'ai commencé à y travailler dans les années 1970. On n'en trouvait guère alors. Je crois donc que la notion de construction de capacités, chère à Amartya Sen, reste plus pertinente et plus importante que jamais. Toutefois, il convient de mesurer ce qu'implique une politique de santé. Autant un instituteur, un professeur, forts de leur savoir et dans un environnement très minimaliste, peuvent transmettre de véritables connaissances, comme on le constate tous les jours dans de nombreux pays, autant l'idée d'un «médecin aux pieds nus» est une chimère qui devrait avoir vécu. Un médecin dépourvu d'équipement diagnostique et thérapeutique ne peut pas soigner. Il peut dispenser de bonnes paroles, mais sans effets pratiques. Les médecins, et les soignants en général, ont besoin de plateaux techniques et d'une structure administrative, sans cela ils n'obtiennent que des résultats ponctuels, certes pas négligeables pour les patients traités, mais qui en termes de santé publique et d'amélioration du niveau sanitaire général sont dérisoires. Les efforts dans le domaine de la construction, de l'équipement, du

1 - Georges Canguilhem (1904-1995), après une thèse de médecine, a contribué fortement au renouvellement de la philosophie des sciences de la vie, et en particulier de la philosophie de la médecine. Il s'est interrogé entre autres sur les limites fluctuantes de la normalité. (NDLR) 
suivi, et donc de la gestion et de l'administration, sont tout aussi importants que les efforts dans le domaine de la recherche clinique et de la mise à disposition des médicaments, sans oublier la continuité de la formation médicale. Les procédures de l'aide internationale et les responsabilités qu'elles engagent se diversifient donc. La mise à disposition de médicaments accessibles d'une part, et l'effort financier indispensable à la construction et à l'entretien des bâtiments et des équipements nécessaires à la mise en ouvre des soins d'autre part : voilà ce qui peut relever de la coopération entre la communauté internationale et les différents États concernés. Quant à la question du prix des médicaments, elle pose en réalité le problème de l'exceptionnalité des produits de santé. On parle d'exception culturelle, pourquoi ne pas parler d'exception sanitaire? La santé est en effet - ou devrait être - un bien public mondial. Elle l'est d'ailleurs objectivement car les maladies infectieuses épidémiques sont transnationales par définition. La nécessité de les traiter ne répond pas seulement aux besoins premiers des malades et aux impératifs de solidarité humaine, elle est aussi un enjeu de sécurité.

TC : Peut-on dire à cet égard quel'Organisation mondiale de la santé (OMS) dispose des moyens nécessaires à une action d'envergure globale ou - et ce n'est pas réduire son rôle - n'est-elle simplement là que pour préconiser de bonnes méthodes, plutôt que de les mettre en ouvre?

R.B. : L'OMS est en effet plutôt là pour préconiser les politiques à suivre. $\mathrm{C}^{\prime}$ est un immense cabinet de conseil qui a théoriquement dans ses statuts une vocation opérationnelle, mais qui dans les faits n'a jamais eu les moyens institutionnels et pratiques pour remplir ce rôle, aujourd'hui révolu. En revanche, la préconisation, la prescription, le cadrage, relèvent de l'OMS, et c'est là une tâche extrêmement importante. Encore faut-il que l'OMS soit indemne d'influences, à commencer par celles des grands laboratoires pharmaceutiques. Regardez la question des génériques. Certes, les médicaments génériques sont préconisés par l'OMS; mais l'organisation ne s'est pas illustrée de manière précise pendant toute la campagne portant sur l'ouverture de la notion de générique et sur la possibilité de déclarer des situations d'exception et d'urgence sanitaire pour pouvoir passer outre les brevets et la propriété intellectuelle, alors que cette bataille a été très importante.

TC : Vous avez souligné combien l'OMS est retenue sur la question de l'urgence sanitaire ou des licences obligatoires. N'est-il pas paradoxal de constater que l'Organisation mondiale du commerce (OMC) s'est davantage engagée dans ce domaine? Après tout, l'OMC reconnaît explicitement que la santé mérite une attention particulière et que tout ne peut pas se régler par le marché. L'organisme chargé de gérer le marché reconnaît mieux la spécificité de la santé que l'Organisation mondiale de la santé. Est-ce parce qu'à l'OMC les pays émergents sont en mesure de faire entendre leur voix plus qu'à OMS, et parce que le rapport force entre les dominants et les dominés est beaucoup plus fluctuant à l'OMC, structure de négociation, que dans les organismes habituels du système onusien?

R.B. : Oui, et c'est la raison pour laquelle l'OMC a pu adopter des mesures que l'OMS ne semblait pas vouloir énoncer. C'est d'ailleurs pourquoi, au moment 
où la contestation de l'OMC était à son comble, je ne partageais pas l'avis des partisans de sa dissolution, parce que j'y voyais au contraire, comme vous venez de le dire, la preuve que c'est une instance de négociation à l'intérieur de laquelle des pays émergents et des voix dissonantes peuvent se faire entendre. Mais il convient d'ajouter que l'OMC a dû faire face à une très forte mobilisation de mouvements sociaux, au sens large du terme, lors de certains de ses sommets. L'OMC ne pouvait pas ignorer ces voix, tandis que l'OMS, elle, n'a pas à subir de telles pressions. Les animateurs des mouvements sociaux pourraient en tirer une leçon et appeler à manifester devant le siège de l'OMS pour l'inciter à résister à l'emprise croissante du marché de la santé. La bonne OMS contre la mauvaise OMC, c'est un schéma qui ne fonctionne pas du tout.

TC : Le concept de crise sanitaire prend aujourd'hui un sens élargi : on y intègre tout à la fois, comme le fait un récent dossier du Journal du CNRS ${ }^{2}$, les maladies virales et les pandémies, les «fléaux silencieux» tels que l'amiante, mais aussi les risques industriels, ceux liés à l'environnement et les périls liés aux évolutions climatiques. Une telle approche vous paraît-elle positive, en ce qu'elle entend couvrir, dans l'analyse comme dans l'action, l'ensemble des facteurs environnementaux, biologiques et socioéconomiques qui mettent en péril la santé publique? Le sentiment diffus d'inquiétude généralisée qu'elle peut contribuer à diffuser est-il une façon de mieux sensibiliser l'opinion au risque et à la nécessité d'agir?

R.B. : Je suis très ambivalent sur cette question. En tant que médecin, j'ai tendance à être très ouvert à la notion élargie de crise sanitaire puisque l'ensemble des secteurs que vous avez mentionnés représente effectivement un risque potentiel pour la santé. Dans l'industrie, et dans bien d'autres domaines, ce risque existe. La complexification très grande des procédés de fabrication des aliments, des procédés de construction ou de certaines productions industrielles nous conduit - nous médecins et institutions sanitaires - à avoir de plus en plus l'œil sur ces secteurs économiques. De ce point de vue, le concept élargi de crise sanitaire me paraît assez pertinent. Cela étant dit, si je suis ambivalent, c'est aussi par ce que je sais qu'avec la peur, on fait passer n'importe quoi. Au nom de l'hygiène et de la prévention des risques d'épidémies, on peut tuer les libertés, restreindre les mouvements et prendre des mesures indéfendables. Il est donc nécessaire de s'inscrire dans une perspective qui ne soit pas uniquement défensive. Il convient par exemple d'apporter les preuves de l'efficacité des mesures prises. Faire de la recherche publique pour surmonter les problèmes, pas pour aiguiser des craintes. Quand on additionne les risques d'origines diverses dont la conjonction peut être dommageable pour la santé, il faut le faire dans un esprit constructif, sinon on risque de nourrir une théologie de la peur, une incitation à la rétraction et une source d'angoisses qui deviendraient elles-mêmes potentiellement pathogènes, ce qui serait un comble! L'équilibre est difficile à trouver, mais il importe de traiter de la notion de crise sanitaire dans un sens encourageant l'action.

2 - Le Journal du CNRS, $\mathrm{n}^{\circ}$ 208, mai 2007. Dossier «Virus, climat, pollution... Menaces sur la santé». Voir l'éditorial de Bernard Delay : "Crises sanitaires : comprendre, prévoir, protéger, guérir», p. 5. 
TC : Ne peut-on dire que le débat actuel sur le principe de précaution relève justement de cette ambivalence? Ceux qui défendent le principe mettent en avant la primauté de la santé publique face à l'incertitude, alors que d'autres considèrent que ce principe tend à paralyser l'innovation. Qu'en pensez-vous?

R.B. : Le principe de précaution est devenu une sorte de syntagme qui pèse lourdement sur les consciences, alors même que la notion de prudence - la prudence au sens grec, la phronèsis, la prudence comme sens de la mesure et non manque d'audace - devrait présider à toutes les actions humaines. Pourquoi introduire sous une formulation très vague cette notion de prudence?

TC : Est-ce une rançon du système démocratique et de la difficulté des responsables politiques à prendre des décisions délicates qui les pousse à s'abriter derrière les avis des experts, parfois divergents?

R.B. : Oui, la constitutionnalisation en France du principe de précaution me semble relever de ce qu'on appelle la démocratie d'opinion, qui pousse à réagir immédiatement face à des préoccupations peut-être parfaitement légitimes, mais qui devraient sans doute susciter d'autres réponses. Naturellement, nous sommes tous sensibles au principe de précaution, en tant que concept et que préoccupation. Mais je crains qu'en l'appuyant sur une argumentation juridique, on prenne le risque de limiter la recherche plutôt que de construire un monde plus sûr.

Entretien conduit par Jean-Luc Racine 